\title{
Wenn die Evidenz fehlt
}

\author{
Urs Brüggera, Alois Gratwohl \\ a Prof. Dr. oec., Winterthurer Institut für Gesundheitsökonomie, Zürcher Hochschule für Angewandte Wissenschaften, Winterthur \\ ${ }^{b}$ Prof. Dr. med., Medizinische Fakultät der Universität Basel
}

Die Literaturangaben finden sich unter

www.saez.ch $\rightarrow$ Aktuelle Ausgabe oder $\rightarrow$ Archiv $\rightarrow 2015 \rightarrow 34$.

"Coverage with evidence development» bietet die Möglichkeit zur vorzeitigen Finanzierung neuer Leistungen, auch wenn die wissenschaftliche Evidenz noch fehlt. Dieses Verfahren wurde nun evaluiert. Neue Ansätze scheinen nötig: Die bisher sequentiellen Schritte von Grundlagenforschung, klinische Forschung, Marktzulassung, Vergütungsentscheid, Anwendung beim Patienten sollten künftig parallel ablaufen. Evidenznachweise sollten global, Finanzierungsentscheide lokal erfolgen.

In einem absolut freien Marktsystem stehen alle medizinischen Leistungen jedem nach Massgabe seiner oder ihrer Wünsche und finanziellen Möglichkeiten zur Verfügung. In einem auf Solidarität basierten Gesundheitssystem ist dies nicht möglich. Die Kluft zwischen potentiell unbegrenzten Wünschen einerseits und limitierten Mitteln anderseits verlangt nach anderen Lösungen. Institutionalisierte Kriterien sollen entscheiden, wem welche medizinischen Leistungen unter welchen Voraussetzungen finanziert werden. Die Schweizer Bevölkerung hat sich für ein solches solidaritätsbasiertes Gesundheitssystem mit einer obligatorischen Grundversicherung entschieden. Das Krankenversicherungsgesetz (KVG) sieht darin vor, dass notwendige medizinische Leistungen übernommen werden, wenn diese wirksam, zweckmässig und wirtschaftlich sind [1]*. Entscheidungen sind einfach, wenn genügend Daten über die Sicherheit, Wirksamkeit, Zweckmässigkeit und Wirtschaftlichkeit vorliegen.

Für einen Grossteil der Leistungen, wie Medikamente, Analysen, Mittel und Gegenstände sowie alle Präven-
Lorsque l'évidence fait défaut

En Suisse, I'assurance de base obligatoire prend normalement en charge toutes les prestations médicales nécessaires, à condition qu'elles soient efficaces, appropriées et économiques. Le système de santé prévoit également des possibilités de financement anticipé de nouvelles prestations prometteuses, même si l'évidence scientifique requise fait encore défaut. Qualifiée de "Coverage with evidence development", cette pratique est assortie de conditions. Entre son introduction en 1996 avec la LAMal et 2013, cette solution a été appliquée pour 33 des 152 évaluations présentées à la Commission fédérale des prestations générales et des principes (CFPP). Ce processus de décision a désormais fait l'objet d'une évaluation scientifique. Les résultats montrent que si la commission est animée de bonnes intentions, les décisions sont fortement influencées par des changements institutionnels. II est donc urgent de prendre des mesures. tionsleistungen gibt es Positivlisten. Das heisst, bezahlt wird nur, was explizit aufgeführt ist. All diese Leistungen werden in einem strukturierten Health Technology Assessment (HTA)-Prozess durch die eidgenössische Kommission überprüft. Anders ist es bei diagnostischen und therapeutischen Leistungen, die durch Ärztinnen und Ärzte ausgeführt werden. Hier gilt das Vertrauensprinzip, dass Ärztinnen und Ärzte grundsätzlich nach diesen Vorgaben handeln. Nur wenn derartige Leistungen umstritten sind, werden sie ebenfalls in einem HTA-Prozess überprüft. Die Eidgenössische Kommission für allgemeine Leistungen und Grundsatzfragen (ELGK) beurteilt, ob Wirksamkeit, Zweckmässigkeit und Wirtschaftlichkeit gegeben sind und gibt dann eine Empfehlung ab, ob und unter welchen Bedingungen eine solche Leistung im Rahmen der obligatorischen Krankenversicherung zu finanzieren ist oder ob sie nicht bezahlt wird. Der Entscheid wird anschliessend vom Eidgenössischen Department des Innern (EDI) gefällt und im Anhang 1 der Krankenpflege-Leistungsverordnung publiziert.

\section{Neue Therapien - umstrittene Leistungen}

Eine Umstrittenheit präsentiert sich immer wieder bei vielversprechenden neuen Therapien oder diagnostischen Massnahmen. Oft liegen erst ungenügende wissenschaftliche Daten vor. Patientinnen und Patienten wünschen bereits bezahlten Zugang zu diesen hoffnungsvollen Möglichkeiten. Ärztinnen und Ärzte möchten die neuen Chancen nutzen können, sie nicht ihren Patienten vorenthalten. Nur, die Evidenz fehlt, dass diese neuen, erfolgsversprechenden Therapien auch sicher, wirksam, zweckmässig und wirtschaftlich sind.

Das KVG hat dieses Dilemma vorhergesehen. Das EDI kann bei positiver Beurteilung durch die Kommission (ELGK) eine solche neue medizinische Leistung trotz 
beschränkter Unterlagen unter Auflagen für eine befristete Zeit in den Leistungskatalog einschliessen. Im Anhang 1 der KLV sind diese Leistungen dann mit dem Vermerk "Ja, in Evaluation» versehen. Die Aufnahme wird mit der Bedingung verbunden, die noch fehlende Evidenz zu erarbeiten. Dieses Verfahren wird als «Coverage with evidence development (CED)» bezeichnet. Dieses ist innovationsfreundlich und orientiert sich primär am Patienten [2]. Es verhindert aber auch, dass unsichere und/oder unwirksame medizinische Leistungen unbenommen und dauernd von der Krankenversicherung bezahlt werden. CED hilft so mit, limitierte Mittel transparent, nachvollziehbar, frühzeitig und patientenzentriert einzusetzen.

\section{Eine Analyse des Verfahrens "Coverage with evidence development (CED)»}

Diese «CED»-Möglichkeit wurde von 1996 bis 2013 bei insgesamt 33 von 152 der ELGK vorgelegten, umstrittenen Leistungen eingesetzt. Dieser Ablauf wurde nun im Rahmen der von der Bangerter-Stiftung und der SAMW-geförderten «Versorgungsforschung Schweiz» erstmals in einer wissenschaftlichen Arbeit retrospektiv analysiert [3]. Gesucht wurde nach Assoziationen zwischen Anzahl und Art der medizinischen Leistungen, der Dauer der «CED»-Periode und der finalen Entscheidung. Geprüft wurden mögliche Zusammenhänge mit den damit verbundenen Auflagen, wie Einschränkung auf bestimmte Institutionen, die Notwendigkeit einer vorgängigen Beurteilung durch den Vertrauensarzt der Krankenversicherung oder die

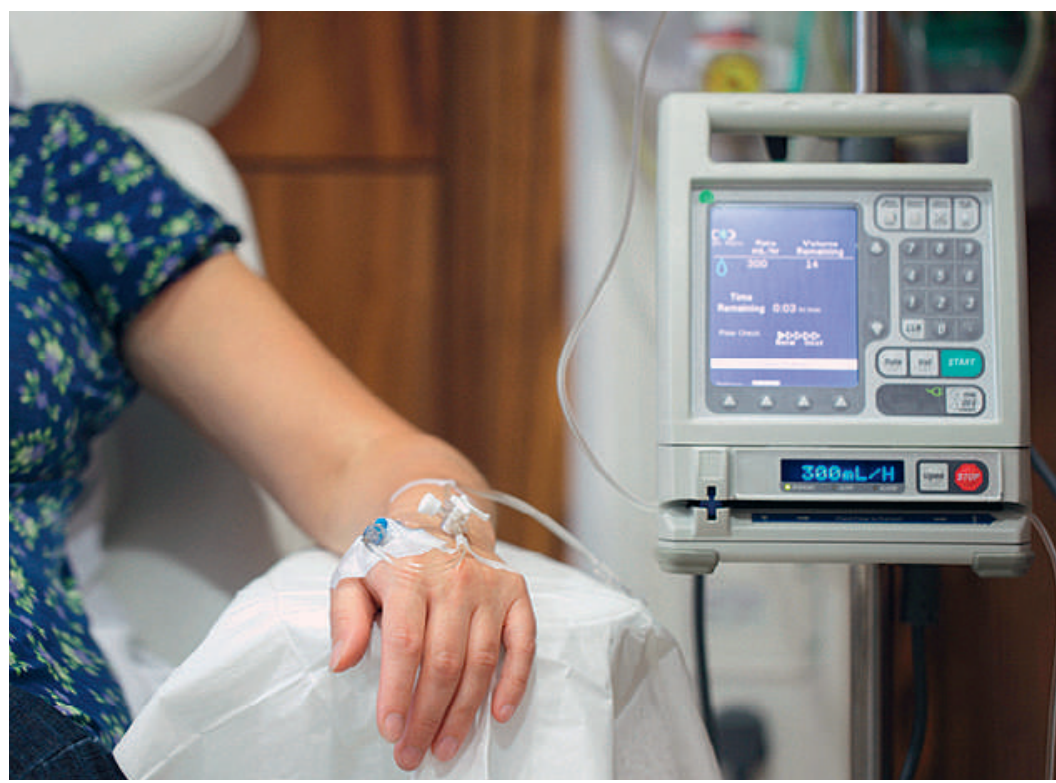

Besonders Tumor-Patienten hoffen auf neue, noch nicht zugelassene Medikamente. Auch hier sind andere Konzepte zur Evidenzentwicklung gefragt als die traditionellen Phase-I-II-III-Verfahren.
Verpflichtung zu Registerführung. Untersucht wurde auch ein möglicher Einfluss institutioneller Veränderungen über die Zeit. Begleitet wurde die Untersuchung durch strukturierte, qualitative Interviews mit ausgewählten Entscheidungsträgern.

Die Resultate waren überraschend und eindeutig. Als einziger signifikanter Faktor erwiesen sich die institutionellen Veränderungen über die Zeit, der Wechsel der Leistungskommission vom Bundesamt für Sozialversicherung zum Bundesamt für Gesundheit, der Wechsel der jeweiligen Departements-Vorsteher oder der Wechsel der Kommissionsvorsitzenden. Die qualitativen Interviews bezeugten den Kommissionsmitgliedern grundsätzlich gute Absichten. Die Interviewten wiesen auch auf die fehlenden wissenschaftlichen Kriterien für die Kommissionsarbeit hin und betonten die Notwendigkeit einer Evaluation ihrer eigenen Arbeit. Diese Ergebnisse rufen nach Veränderungen. Guter Wille in der Kommission allein genügt nicht mehr. Das BAG hat in der Folge seit Herbst 2014 eine neue Check-

Die Eidg. Leistungskommission und das Medical Board in ihren Funktionen und ihrer Zusammensetzung müssen überdacht werden.

liste erarbeitet; damit werden einige der bisherigen Mängel verbessert [4]. Aber auch das genügt noch nicht. Zudem wird das Bedürfnis nach «CED» steigen. Gerade im Rahmen der Personalisierten Medizin, oder precision medicine verläuft die Entwicklung unglaublich schnell. Neue Targets werden entdeckt, neue zielgerichtete Substanzen entwickelt. Ärztinnen und Ärzte möchten diese «sofort» einsetzen, betroffene Patientinnen und Patienten diese "sofort" erhalten. Die Verfahren sind oft sehr teuer und die Studienlage zur wissenschaftlichen Evidenz ist dünn. Doch andere Konzepte zur Evidenzentwicklung sind hier gefragt als die traditionellen Phase-I-II-III-Verfahren [5]. Die Zeitspanne zwischen Entwicklung und routinemässiger Anwendung in der Gesundheitsversorgung, als second gap in translation bezeichnet, ist jetzt schon zu lang [6]

\section{Paralleles statt sequentielles Vorgehen}

Wir plädieren für grundsätzlich neue Ansätze. Die bisher getrennten Schritte im Prozess von der Grundlagenforschung zur klinischen Forschung und zur Marktzulassung, von dieser zum Vergütungsentscheid und Anwendung beim Patienten müssen zukünftig parallel und nicht mehr strikt sequentiell ablaufen. Zulassungsbehörden für Medikamente wie die European Medicines Agency (EMA) haben diese Problematik erkannt. Die EMA spricht von adaptive pathways. Sie will, dass auch die Marktzulassung schneller, aber mit Auf- 
lagen zur weiteren Evidenzgenerierung abläuft [7]. CED auf der Stufe der Vergütungsentscheide ist ein gleichwertiger Ansatz, der verschiedene Schritte parallelisiert. Voraussetzungen dazu sind präzise Fragestellungen und definierte Bedingungen für die anschliessend notwendigen Studien oder Register. Dieses Fehlen von Kriterien zu Beginn eines CED-Prozesses zeigte sich als eine schwerwiegende Schwäche, wie in der Studie gezeigt in der Schweiz, aber auch in anderen Ländern. Wenn bei dieser neuen Form von CED die Generierung von wissenschaftlicher Evidenz und die politische Entscheidung näher zusammenrücken, zeitlich nicht mehr klar getrennt sind, birgt dies zusätzliche Gefahren. Risiken für Fehlentscheide sind immanent in jedem HTA-Prozess. Das jüngste Strategiepapier des Health Technology Assessment Network der Europäischen Union zu Health Technology Assessment (HTA) nimmt diese Sorgen auf und gibt eine klare Vorgabe: evidence is global-decisions are local [8].

\section{Evidenznachweis global - Finanzierungs- entscheid lokal}

Die Aussage präzisiert und bezeichnet die Evaluation der medizinischen Daten, das heisst das Erarbeiten der Evidenz als eine wissenschaftliche Aufgabe. Die Evidenz zu einer bestimmten Technologie wird dabei immer unabhängig vom lokalen Kontext ausfallen. Die Entscheidung umgekehrt, ob eine bestimmte medizinische Leistung übernommen und finanziert werden soll, ist eine politische Aufgabe. Der Entscheid kann je nach den Bedürfnissen und Möglichkeiten in jedem Land anders ausfallen und hängt vom lokalen Umfeld ab. Diese unterschiedlichen Aufgaben, Beurteilung der Evidenz und Beschluss über Finanzierung müssen getrennt werden. Dies gilt umso mehr bei CED, wenn beide Prozesse parallel stattfinden. Der Leitsatz gilt dabei nicht nur für neue medizinische Leistungen, sondern ebenso für die Überprüfung bisheriger, eventuell überholter Leistungen. Dieses als disinvestment bezeichnete Verfahren sollte nach den gleichen Kriterien verlaufen.

Was heisst das für die Schweiz? HTA wurde bisher in der Schweiz eher als bürokratischer Prozess, nicht als akademische Herausforderung betrachtet; die Notwendigkeit dafür in einem föderalistischen System und in einem reichen Land wie der Schweiz eher geringgeschätzt. Die verantwortlichen Institutionen im Bereich HTA Schweiz sollten deshalb die Konzepte des EU-Strategiepapiers aufnehmen und neue, hoffnungsvolle medizinische Verfahren wie auch alte umstrittene Leistungen nach gleichem Muster überprüfen. Die Eidgenössische Leistungskommission und das Medical Board in ihren Funktionen und ihrer Zusammensetzung müssen überdacht werden.
Die Prozesse sind zu überprüfen. Für die geforderte Methodik zur Beurteilung der Evidenz braucht es Fachwissen und klare Guidelines. Die Universitäten und Fachgesellschaften sind gefordert, den Rahmen für eine wissenschaftliche Evidenzbeurteilung vorzubereiten und entsprechende Kapazitäten und Know-how aufzubauen. Wenn die Evidenz mangelhaft ist, darf die Entscheidung über «Evidenz» nicht einem politischen Gremium überlassen werden. Mammascreening wäre ein exemplarisches Beispiel, wo Politik über Wissen regiert. Die Schweiz könnte zudem bei fraglicher Evidenz modellartig Pionierarbeit leisten. Medizinische Fachgesellschaften könnten für die zu prüfenden Leistungen (unabhängig, ob neu und vielversprechend oder alt und angezweifelt) vergleichende Studien vorschlagen. Die zu prüfende neue medizinische Massnahme wird mit dem bisherigen Standard, die fraglich zu verlassende alte Massnahme mit der neu vorgeschlagenen Methodik in einer prospektiven Studie verglichen. Eine solche Studie beinhaltet Kosten. Die Finanzierung der Studienkosten soll wie die der Behandlungskosten über den obligatorischen Kostenträger erfolgen. Voraussetzung dafür ist ein Entscheid der ELGK für CED. Dies ist nach heutigem Stand des KVG möglich. Datenanalyse und Datenevaluation sind integrale Bestandteile einer Therapie; Qualitätskontrollen sind im KVG vorgesehen. Der Vergleich mit der «Standardbehandlung» dient dabei der Qualitätskontrolle und der Evidenzerarbeitung. Für die Übernahme der Leistung als Pflichtleistung wäre die Teilnahme bei CED-Erhebungen obligatorisch. Die Freiwilligkeit der Patienten bleibt dabei gewahrt.

Wir sind klar der Meinung: Solidarität beinhaltet Rechte und Pflichten. Das Recht auf Nichtteilnahme unter Eigenbezahlung bleibt bestehen; nur bei Anspruch auf Bezahlung folgt die Pflicht zur Studienteilnahme. Über diesen Punkt herrscht in der Schweiz kein Konsens; eine öffentliche Diskussion dazu ist notwendig. Idealerweise wird das Forschungskonzept bei Schweizer CED-Studien in internationale Evaluationen integriert; zudem können dann gleichzeitig Daten für Kosten-Nutzen-Analysen in der Schweiz und für die Schweiz generiert werden. Die Evaluation erfolgt nach wissenschaftlichen Kriterien. Der Entscheid über Aufnahme in den Leistungskatalog, oder Ablehnung, bleibt den politischen Instanzen vorbehalten. Die Politik, das Bundesamt für Gesundheit und die medizinischen Verantwortungsträger sind gefordert.

Interessenkonflikt

Die Studie wurde zum Teil von der Bangerter-Stiftung unterstützt. Die Arbeit liegt alleine in der Verantwortung der Autoren.

Bildnachweis

(c) Flynt/Dreamstime.com 


\section{Referenzen}

1 www.bag.admin.ch/themen/krankenversicherung (Mai 4 2015).

2 Menon D, McCabe CJ, Stafinski T, et al. Principles of design of access with evidence development approaches: a consensus statement from the Banff Summit. PharmacoEconomics 2010;28(2):109-11.

3 Brügger U, Horisberger B, Ruckstuhl A, Plessow R, Eichler K, Gratwohl A. Health technology assessment in Switzerland: a descriptive analysis of "Coverage with Evidence Development" decisions from 1996 to 2013. BMJ Open. 2015; Mar 27;5(3):e007021.

4 www.bag.admin.ch/themen/

krankenversicherung/00263/00264/04853/index.html?lang=de
5 Husereau D, Marshall DA, Levy AR, et al. Health technology assessment and personalized medicine: are economic evaluation guidelines sufficient to support decision making? International journal of technology assessment in health care. 2014;30(2):179-87.

6 Cooksey D. A review of UK health research funding. Secondary A review of UK health research funding 2006. www.gov.uk/ government/uploads/system/uploads/attachment_data/ file/228984/0118404881.pdf

7 www.ema.europa.eu/ema/index.jsp?curl=pages/regulation/ general/general_content_000601.jsp

8 http://ec.europa.eu/health/technology_assessment/policy/ network/index_en.htm 